ANNALES

POLONICI MATHEMATICI

LXXVIII.2 (2002)

\title{
Recurrent point set of the shift on $\Sigma$ and strong chaos
}

\author{
by LiDONG WANG (Dalian), \\ Gongfu LiaO (Changchun) and YU Yang (Siping)
}

\begin{abstract}
Let $(\Sigma, \varrho)$ be the one-sided symbolic space (with two symbols), and let $\sigma$ be the shift on $\Sigma$. We use $A(\cdot), R(\cdot)$ to denote the set of almost periodic points and the set of recurrent points respectively. In this paper, we prove that the one-sided shift is strongly chaotic (in the sense of Schweizer-Smítal) and there is a strongly chaotic set $\mathcal{J}$ satisfying $\mathcal{J} \subset R(\sigma)-A(\sigma)$.
\end{abstract}

1. Introduction. Throughout this paper, $X$ will denote a compact metric space with metric $d ; I$ is the closed interval $[0,1]$.

For a continuous map $f: X \rightarrow X$, denote the sets of periodic points, nonwandering points and $\omega$-limit points of $f$ by $P(f), \Omega(f)$ and $\omega(f)$ respectively; $f^{n}$ will denote the $n$-fold iterate of $f$.

$D \subset X$ is said to be a chaotic set of $f$ if for any different points $x, y \in D$,

$$
\liminf _{n \rightarrow \infty} d\left(f^{n}(x), f^{n}(y)\right)=0, \quad \limsup _{n \rightarrow \infty} d\left(f^{n}(x), f^{n}(y)\right)>0 .
$$

$f$ is said to be chaotic if it has a chaotic set which is uncountable.

The notion of strong chaos first occurred in [18] where it is characterised by the distribution function of distances between trajectories of two points. The concrete version is as follows.

Let $x, y \in X$. For any real $t>0$, let

$$
\begin{aligned}
F_{x y}(t) & =\liminf _{n \rightarrow \infty} \frac{1}{n} \sum_{i=1}^{n} \chi_{[0, t)}\left(d\left(f^{i}(x), f^{i}(y)\right)\right), \\
F_{x y}^{*}(t) & =\limsup _{n \rightarrow \infty} \frac{1}{n} \sum_{i=1}^{n} \chi_{[0, t)}\left(d\left(f^{i}(x), f^{i}(y)\right)\right),
\end{aligned}
$$

where $\chi_{A}$ is the characteristic function of the set $A$. Obviously, $F_{x y}$ and

2000 Mathematics Subject Classification: 58F13, 58F20.

Key words and phrases: recurrent point, shift, strong chaos.

Project supported by the National Natural Science Foundation of China. 
$F_{x y}^{*}$ are both nondecreasing functions; for $t \leq 0$, define $F_{x y}$ and $F_{x y}^{*}$ as probability distribution functions (see [18] for details, where $F_{x y}$ and $F_{x y}^{*}$ are called, respectively, the lower and upper distribution function of $x$ and $y$ ).

Call $x, y \in X$ a pair of points displaying strong chaos if

1) $F_{x y}^{*}=\chi_{[0, \infty)}$, i.e., $F_{x y}^{*}(t)=1$ for all $t>0$,

2) $F_{x y}(\varepsilon)=0$ for some $\varepsilon>0$.

$f$ is said to display strong chaos if there exists an uncountable set $D \subset X$ such that any two different points in $D$ display strong chaos.

Clearly any map displaying strong chaos must be Li-Yorke chaotic.

For a continuous map $f: I \rightarrow I, \mathrm{Li}$ and Yorke [10] has proved that if $f$ has a periodic point of period 3 , then it is chaotic.

Later, many sharpened results came into being in succession (see [7]-[9], [11], [13], [16], [17], [19]). One can find in [11] and [1] equivalent conditions for $f$ to be chaotic, and in [16] or [19] a chaotic map with topological entropy zero, which has shown that positive topological entropy and chaos are not equivalent. On the other hand, it is known that by restricting the uncountable chaotic set to $R(f)$ or to $\overline{P(f)}$ or to $\Omega(f)$, the equivalence holds (see [4], [20], [21]).

This leaves open two questions:

1) Is the existence of an uncountable strongly chaotic set of $f$ in $R(f)$ or in $A(f)$ equivalent to $\operatorname{ent}(f)>0$ ?

2) Is there a map $f$ such that $R(f)-A(f)$ contains an uncountable strongly chaotic set?

For a continuous map $f: I \rightarrow I$, Schweizer and Smítal [18] have proved:

(i) If $f$ has zero topological entropy, then no pair of points can form a strongly chaotic set. (This implies that strong chaos and Li-Yorke chaos are not the same notion.)

(ii) If $f$ has positive entropy, then there exists an uncountable strongly chaotic set in which each member is an $\omega$-limit point of $f$.

The aim of this paper is to learn whether there exists a map $f$ such that $R(f)-A(f)$ contains an uncountable strongly chaotic set of $f$.

In fact, we will prove

THEOREM. The one-sided shift is strongly chaotic and there is a strongly chaotic set $\mathcal{J}$ satisfying $\mathcal{J} \subset R(\sigma)-A(\sigma)$.

2. Basic definitions and preparations. Let $S=\{0,1\}, \Sigma=\{x=$ $\left.x_{1} x_{2} \ldots \mid x_{i} \in S, i=1,2, \ldots\right\}$ and define $\varrho: \Sigma \times \Sigma \rightarrow \mathbb{R}$ as follows: for any 
$x, y \in \Sigma$, if $x=x_{1} x_{2} \ldots$ and $y=y_{1} y_{2} \ldots$, then

$$
\varrho(x, y)=\left\{\begin{array}{ll}
0 & \text { if } x=y, \\
1 / 2^{k} & \text { if } x \neq y,
\end{array} \text { and } \quad k=\min \left\{n \mid x_{n} \neq y_{n}\right\}-1 .\right.
$$

It is not difficult to check that $\varrho$ is a metric on $\Sigma$. The space $(\Sigma, \varrho)$ is compact and called the one-sided symbolic space.

Define $\sigma: \Sigma \rightarrow \Sigma$ by $\sigma(x)=x_{2} x_{3} \ldots$ for any $x=x_{1} x_{2} \ldots \in \Sigma$. Then $\sigma$ is continuous and called the shift on $\Sigma$. Call $A$ a tuple (over $S$ ) if it is a finite arrangement of elements in $S$. If $A=a_{1} a_{2} \ldots a_{n}$ where $a_{i} \in S, 1 \leq i \leq n$, then the length of $A$ is said to be $n$, denoted by $|A|=n$.

Let $B=b_{1} b_{2} \ldots b_{m}$ be another tuple. Set $A B=a_{1} a_{2} \ldots a_{n} b_{1} b_{2} \ldots b_{m}$; then $A B$ is also a tuple.

Let $x \in \Sigma$ with $x=x_{1} x_{2} \ldots$. It is called a repeating sequence with recurring period of length $m$ if $x_{i+m}=x_{i}$ for any $i \in\{1,2, \ldots\}$. We then write $x=\left(\dot{x}_{1} \dot{x}_{2} \ldots \dot{x}_{m}\right)$.

Let $x \in X$. Then $y \in X$ is said to be an $\omega$-limit point of $x$ if the sequence $f(x), f^{2}(x), \ldots$ has a subsequence converging to $y$. The set of $\omega$-limit points of $x$ is denoted by $\omega(x, f)$. Each point in $\bigcup_{x \in X} \omega(x, f)$ is called an $\omega$-limit point of $f$. The set of $\omega$-limit points of $f$ is denoted by $\omega(f)$.

$x \in X$ is called almost periodic for $f$ if for any $\varepsilon>0$, one can find $k>0$ such that for any integer $q \geq 0$, there is an integer $r$ with $q \leq r<k+q$ satisfying $d\left(f^{r}(x), x\right)<\varepsilon$. Denote by $A(f)$ the set of almost periodic points of $f$.

$x \in X$ is called a recurrent point for $f$ if the sequence $f(x), f^{2}(x), \ldots$ has a subsequence converging to $x$. The set of recurrent points for $f$ is denoted by $R(f)$.

$Y \subset X$ is called a minimal set of $f$ if for any $x \in Y, \omega(x, f)=Y$.

Lemma 2.1. For any $x \in X$ and any $N>0$, the following are equivalent.

1) $x \in A(f)$.

2) $x \in A\left(f^{N}\right)$.

3) $x \in \omega(x, f)$ and $\omega(x, f)$ is a minimal set of $f$.

For a proof see [5] and [6].

LEMMA 2.2. $\Sigma$ has an uncountable subset $E$ such that for any different points $x=x_{1} x_{2} \ldots, y=y_{1} y_{2} \ldots$ in $E, x_{n}=y_{n}$ for infinitely many $n$ and $x_{m} \neq y_{m}$ for infinitely many $m$.

Proof. For any $x=x_{1} x_{2} \ldots, y=y_{1} y_{2} \ldots \in \Sigma$, write $x \sim y$ if either $x_{n}=y_{n}$ holds only for finitely many $n$, or $x_{m} \neq y_{m}$ holds only for finitely many $m$. We easily check that $\sim$ is an equivalence relation on $\Sigma$. Let $x \in \Sigma$. It is easy to see that the set $\{y \in \Sigma \mid y \sim x\}$ is countable and so the quotient 
set $\Sigma / \sim$ is uncountable. Taking a representative in each equivalence class of $\Sigma / \sim$, we get an uncountable set $E$ which satisfies the requirement.

LEMMA 2.3. Let $(\Sigma, \varrho)$ be the one-sided symbolic space, and let $\sigma$ be the shift on $\Sigma$. Then:

(i) For all $s \in \Sigma$ and $m>0, \sigma^{m}(s)=s$ if and only if $s=\left(\dot{s}_{0} \dot{s}_{1} \ldots \dot{s}_{m-1}\right)$ (i.e. $s$ is a repeating sequence with recurring period of length $m$ ).

(ii) There are exactly $2^{n}$ elements $s$ in $\Sigma$ such that $\sigma^{n}(s)=s$.

For a proof see [3].

3. Proof of the Theorem. First we construct the set $\mathcal{J}$ as in the conclusion of the Theorem.

Let $A=a_{1} a_{2} \ldots a_{n}$ be a tuple (over $S=\{0,1\}$ ). Define the inverse of $A$ to be $\bar{A}=\bar{a}_{1} \ldots \bar{a}_{n}$, where

$$
\bar{a}_{i}=\left\{\begin{array}{ll}
0 & \text { if } a_{i}=1, \\
1 & \text { if } a_{i}=0,
\end{array} \quad \text { for } i=1, \ldots, n\right.
$$

Clearly, $|\bar{A}|=|A|$ and $\overline{\bar{A}}=A$.

Take an arbitrary tuple $A_{1}$. Let $A_{2}$ be an arrangement of $A_{1}$ and $\bar{A}_{1}$, say $A_{2}=A_{1} \bar{A}_{1}$ (or $\bar{A}_{1} A_{1}$ ). Define inductively the tuples $A_{2}, A_{3}, \ldots$ such that $A_{n}$ is an arrangement of all the tuples of the finite set

$$
\mathcal{J}_{n}=\left\{J_{1} \ldots J_{n-1} \mid J_{i} \in\left\{A_{i}, \bar{A}_{i}\right\}, 1 \leq i \leq n-1\right\} .
$$

For each $n=1,2, \ldots$, put $m_{n}=\left|A_{1} A_{2} \ldots A_{n}\right|$. Then $m_{n}-m_{n-1}=$ $2^{n-1} m_{n-1}$ for all $n>1$, as can be easily derived from the definition.

Choose an uncountable subset $E$ in $\Sigma$ such that for any different points $x=x_{1} x_{2} \ldots, y=y_{1} y_{2} \ldots$, both $x_{n}=y_{n}$ holds for infinitely many $n$ and $x_{m} \neq y_{m}$ holds for infinitely many $m$. By Lemma 2.2, such a subset exists. Define $\varphi: E \rightarrow E$ by $\varphi(x)=J_{1} J_{2} \ldots=\left\langle J_{i}\right\rangle, i=1,2, \ldots$ for all $x=$ $x_{1} x_{2} \ldots \in E$, where

$$
J_{i}=\left\{\begin{array}{ll}
A_{i} & \text { if } x_{i}=1, \\
\bar{A}_{i} & \text { if } x_{i}=0,
\end{array} \quad \text { for } i=1,2, \ldots\right.
$$

Let $D=\varphi(E)$; then $D \subset E$. Since $E$ is uncountable and $\varphi$ is injective, $D$ is uncountable.

By Lemma 2.3, for any $n \in \mathbb{N}$, the elements of $\Sigma$ satisfying $\sigma^{n}(s)=s$ are the repeating sequences with recurring period of length $n$; there are exactly $2^{n}$ such elements.

Let $p_{1}$ be an arrangement of the recurring periods of the two repeating sequences of length 2 such that $\sigma^{2}(s)=s$, e.g. $p_{1}=01$.

Let $p_{2}$ be an arrangement of the recurring periods of the $2^{2}$ repeating sequences of length 2 such that $\sigma^{2}(s)=s$, e.g. $p_{2}=00011011$. 
$p_{n}$ is an arrangement of the recurring periods of the $2^{n}$ repeating sequences of length $n$ such that $\sigma^{n}(s)=s$, e.g. $p_{n}=000 \ldots 0 \ldots 11 \ldots 1$.

Let $a=p_{1} p_{2} \ldots p_{n} \ldots=0100011011000 \ldots 001 \ldots=a_{1} a_{2} \ldots$ It is easy to see that $\omega(a, \sigma)=\Sigma$. In fact, for any $x=x_{1} x_{2} \ldots \in \Sigma$, let $T_{n}$ be a periodic point of $\sigma$ with period $n$ and with recurring period $\left(x_{1}, \ldots, x_{n}\right)$. Then $T_{n} \rightarrow x(n \rightarrow \infty)$. By the construction of $a$, for any $\varepsilon>0$, there exists $N_{i}(\varepsilon)$ such that

$$
\left|\sigma^{N_{i}}(a)-x\right|<\sum_{n=N_{i}}^{\infty} \frac{1}{2^{n}}<\varepsilon .
$$

So, $\sigma^{N_{i}}(a) \rightarrow x(i \rightarrow \infty)$. This shows $x \in \omega(a, \sigma)$, i.e. $\Sigma \subset \omega(a, \sigma)$. On the other hand, $\omega(a, \sigma) \subset \Sigma$, hence $\Sigma=\omega(a, \sigma)$. Let

$$
\mathcal{J}=\left\{J_{1} a_{1} J_{2} a_{1} a_{2} \ldots J_{n-1} a_{1} a_{2} \ldots a_{n-1} J_{n} \ldots \mid\left\langle J_{i}\right\rangle \in D\right\} .
$$

Since $D$ is uncountable, $\mathcal{J}$ is uncountable, and for any $x, y \in \mathcal{J}$, by Lemma 2.2 we have $x \neq y$.

Secondly, we prove $\mathcal{J} \subseteq R(\sigma)-A(\sigma)$.

Since $\omega(a, \sigma)=\Sigma$, for any $x \in \Sigma$, there exists an infinite sequence $\left\{p_{i}\right\}$ of positive integers such that $\sigma^{p_{i}}(a) \rightarrow x(i \rightarrow \infty)$. For any $C \in \mathcal{J}$, $C=C_{1} a_{1} C_{2} a_{1} a_{2} \ldots$, put

$$
q_{i}=p_{i}+m_{p_{i}}+\frac{\left(p_{i}-1\right)\left(p_{i}-2\right)}{2} .
$$

Then $\sigma^{q_{i}}(C) \rightarrow x(i \rightarrow \infty)$.

Thus we have proved that $\omega(C, \sigma)=\Sigma$ and $C \in R(\sigma)$. Since $\omega(C, \sigma)=\Sigma$ and $\Sigma$ is not a minimal set of $f$, we know that $\omega(C, \sigma)$ is not a minimal set of $f$. By Lemma 2.1, $C \notin A(\sigma)$. Summing up, we obtain

$$
\mathcal{J} \subset R(\sigma)-A(\sigma) .
$$

Finally, we will prove that $\left.\sigma\right|_{\mathcal{J}}$ displays strong chaos.

Let $b=B_{1} a_{1} B_{2} a_{1} a_{2} \ldots$ and $c=C_{1} a_{1} C_{2} a_{1} a_{2} \ldots$ be different points in $\mathcal{J}$, where $B_{i}, C_{i} \in\left\{A_{i}, \bar{A}_{i}\right\}, i=1,2, \ldots$ By Lemma 2.2 and the construction of $\mathcal{J}$, there are sequences of positive integers $p_{i} \rightarrow \infty$ and $q_{i} \rightarrow \infty$ such that

$$
B_{p_{i}}=C_{p_{i}}, \quad B_{q_{i}}=\bar{C}_{q_{i}} \text { for all } i .
$$

Put, for simplicity,

$$
\delta_{b c}(j)=\varrho\left(\sigma^{j}(b), \sigma^{j}(c)\right), \quad j=1,2, \ldots
$$

First, it is easily seen that for given $p_{i} \geq 3$, the first $m_{p_{i}-1}$ symbols of $\sigma^{j}(b)$ 
and $\sigma^{j}(c)$ coincide for

$$
\begin{aligned}
m_{p_{i}-1}+\frac{\left(p_{i}-2\right)\left(p_{i}-3\right)}{2} \leq j \leq & \left(m_{p_{i}}+\frac{p_{i}\left(p_{i}-1\right)}{2}\right) \\
& -\left(m_{p_{i}-1}+\frac{\left(p_{i}-2\right)\left(p_{i}-3\right)}{2}\right),
\end{aligned}
$$

so

$$
\delta_{b c}(j) \leq \frac{1}{2^{m_{p_{i}-1}}} .
$$

Thus $\delta_{b c}(j)<t$ for given $t>0$, provided $p_{i}$ is large enough. Furthermore $\chi_{[0, t)}\left(\delta_{b c}(j)\right)=1$. Let

$$
\begin{aligned}
N_{p_{i}} & =m_{p_{i}}+\frac{\left(p_{i}-1\right) p_{i}}{2}-\left(m_{p_{i}-1}+\frac{\left(p_{i}-2\right)\left(p_{i}-3\right)}{2}\right), \\
K_{p_{i}} & =m_{p_{i}-1}+\frac{\left(p_{i}-2\right)\left(p_{i}-3\right)}{2} .
\end{aligned}
$$

Thus, we have

$$
\frac{1}{N_{p_{i}}} \sum_{j=1}^{N_{p_{i}}} \chi_{[0, t)}\left(\delta_{c b}(j)\right) \geq \frac{1}{N_{p_{i}}} \sum_{j=K_{p_{i}}}^{N_{p_{i}}} \chi_{[0, t)}\left(\delta_{c b}(j)\right)
$$

and

$$
\begin{aligned}
\frac{N_{p_{i}}-K_{p_{i}}}{N_{p_{i}}} & =1-\frac{m_{p_{i}-1}+\frac{\left(p_{i}-2\right)\left(p_{i}-3\right)}{2}}{\left(m_{p_{i}}+\frac{\left(p_{i}-1\right) p_{i}}{2}\right)-\left(m_{p_{i}-1}+\frac{\left(p_{i}-2\right)\left(p_{i}-3\right)}{2}\right)} \\
& =1-\frac{m_{p_{i}-1}+\frac{\left(p_{i}-2\right)\left(p_{i}-3\right)}{2}}{2^{p_{i}-1} m_{p_{i}-1}+\frac{\left(p_{i}-1\right) p_{i}-\left(p_{i}-2\right)\left(p_{i}-3\right)}{2}} \\
& \rightarrow 1 \quad\left(p_{i} \rightarrow \infty\right) .
\end{aligned}
$$

Hence

$$
F_{b c}^{*}(t)=1 .
$$

Secondly, it is easy to see that for given $q_{i}$ large enough, the first $m_{q_{i}-1}$ symbols of $\sigma^{j}(b)$ and $\sigma^{j}(c)$ are all distinct for

$$
m_{q_{i}-1}+\frac{\left(q_{i}-1\right)\left(q_{i}-2\right)}{2} \leq j \leq m_{q_{i}}-m_{q_{i}-1}+\frac{\left(q_{i}-1\left(q_{i}-2\right)\right.}{2},
$$

so

$$
\delta_{b c}(j)=1
$$


Take any $t \in(0,1]$; then $\chi_{[0, t)}\left(\delta_{b c}(j)\right)=0$. Furthermore let

$$
T_{q_{i}}=\left(m_{q_{i}}+\frac{\left(q_{i}-1\right)\left(q_{i}-2\right)}{2}\right)-\left(m_{q_{i}-1}+\frac{\left(q_{i}-1\right)\left(q_{i}-2\right)}{2}\right) .
$$

Then

$$
\begin{aligned}
\sum_{j=1}^{T_{q_{i}}} \chi_{[0, t)}\left(\delta_{b c}(j)\right) & \leq \frac{1}{m_{q_{i}}-m_{q_{i}-1}} \sum_{j=1}^{m_{q_{i}-1}} \chi_{[0, t)}\left(\delta_{b c}(j)\right) \\
& \leq \frac{m_{q_{i}-1}}{2^{q_{i}-1} m_{q_{i}-1}} \rightarrow 0 \quad\left(q_{i} \rightarrow \infty\right) .
\end{aligned}
$$

This shows

$$
F_{b c}(t)=0 .
$$

(1) and (2) prove that $b$ and $c$ are a pair of points displaying strong chaos. By the arbitrariness of $b$ and $c,\left.\sigma\right|_{\mathcal{J}}$ displays strong chaos.

The proof of the Theorem is complete.

\section{References}

[1] L. S. Block and W. A. Coppel, Dynamics in One Dimension, Lecture Notes in Math. 1513, Springer, Berlin, 1992.

[2] E. M. Coven and Z. Nitecki, Nonwandering sets of the powers of maps of the interval, Ergodic Theory Dynam. Systems 1 (1981), 9-31.

[3] R. L. Devaney, An Introduction to Chaotic Dynamical Systems, 2nd ed., AddisonWesley, Redwood City, CA, 1989.

[4] B. S. Du, Every chaotic interval map has a scrambled set in the recurrent set, Bull. Austral. Math. Soc. 39 (1989), 259-264.

[5] P. Erdős and A. H. Stone, Some remarks on almost periodic transformations, Bull. Amer. Math. Soc. 51 (1945), 126-130.

[6] W. H. Gottschalk, Orbit-closure decompositions and almost periodic properties, ibid. 50 (1944), 915-919.

[7] K. Janková and J. Smítal, A characterization of chaos, Bull. Austral. Math. Soc. 34 (1986), 283-292.

[8] T. Y. Li, M. Misiurewicz, G. Pianigiani and J. Yorke, Odd chaos, Phys. Lett. A 87 (1982), 271-273.

[9] - , —, - - No division implies chaos, Trans. Amer. Math. Soc. 273 (1982), 191-199.

[10] T. Y. Li and J. A. Yorke, Period 3 implies chaos, Amer. Math. Monthly 82 (1975), 985-992.

[11] G. F. Liao, Chain recurrent orbits of mapping of the interval, Northeastern Math. J. 2 (1986), 240-244.

[12] -, A note on a chaotic map with topological entropy 0, ibid. 2 (1986), 379-382.

[13] -, $\omega$-limit and minimal sets for maps of the interval, ibid. 5 (1989), 485-489.

[14] - $-\omega$-limit sets and chaos for maps of the interval, ibid. 6 (1990), 127-135.

[15] G. F. Liao and L. Y. Wang, Almost periodicity, chain recurrence and chaos, Israel J. Math. 93 (1996), 145-156. 
[16] M. Misiurewicz and J. Smítal, Smooth chaotic maps with zero topological entropy, Ergodic Theory Dynam. Systems 8 (1988), 421-424.

[17] Y. Oono, Period $\neq 2^{n}$ implies chaos, Progr. Theoret. Phys. F 59 (1978), 1028-1030.

[18] B. Schweizer and J. Smítal, Measures of chaos and a spectral decomposition of dynamical systems on the interval, Trans. Amer. Math. Soc. 344 (1994), 737-754.

[19] J. C. Xiong, A chaotic map with topological entropy 0, Acta Math. Sci. 6 (1986), 439-443.

[20] R. S. Yang, Pseudo-shift-invariant sets and chaos, Chinese Ann. Math. Ser. A 13 (1992), 22-25 (in Chinese).

[21] Z. L. Zhou, Chaos and topological entropy, Acta Math. Sinica 31 (1988), 83-87 (in Chinese).

[22] —, Symbolic Dynamics, Shanghai Scientific and Technological Education Publ. House, Shanghai, 1997.

Department of Mathematics

Dalian University for National Minorities

Dalian 116600, P.R. China

Department of Computer Science

Siping Teacher's College

Siping 136000, P.R. China
Department of Mathematics Jilin University Changchun 130023, P.R. China E-mail: liaogf@public.cc.jl.cn

Reçu par la Rédaction le 18.8.2000

Révisé le 20.6.2001 